Article

\title{
Fractional Schrödinger Equation in the Presence of the Linear Potential
}

\author{
André Liemert* and Alwin Kienle \\ Institut für Lasertechnologien in der Medizin und Meßtechnik an der Universität Ulm, Helmholtzstr. 12, \\ D-89081 Ulm, Germany; alwin.kienle@ilm-ulm.de \\ * Correspondence: andre.liemert@ilm-ulm.de; Tel.: +49-731-1429-223
}

Academic Editor: Rui A. C. Ferreira

Received: 24 March 2016; Accepted: 21 April 2016; Published: 4 May 2016

Abstract: In this paper, we consider the time-dependent Schrödinger equation:

$$
i \frac{\partial \psi(x, t)}{\partial t}=\frac{1}{2}(-\Delta)^{\frac{\alpha}{2}} \psi(x, t)+V(x) \psi(x, t), \quad x \in \mathbb{R}, \quad t>0
$$

with the Riesz space-fractional derivative of order $0<\alpha \leq 2$ in the presence of the linear potential $V(x)=\beta x$. The wave function to the one-dimensional Schrödinger equation in momentum space is given in closed form allowing the determination of other measurable quantities such as the mean square displacement. Analytical solutions are derived for the relevant case of $\alpha=1$, which are useable for studying the propagation of wave packets that undergo spreading and splitting. We furthermore address the two-dimensional space-fractional Schrödinger equation under consideration of the potential $V(\boldsymbol{\rho})=\mathbf{F} \cdot \boldsymbol{\rho}$ including the free particle case. The derived equations are illustrated in different ways and verified by comparisons with a recently proposed numerical approach.

Keywords: Riesz fractional derivative; Caputo fractional derivative; Mittag-Leffler matrix function; fractional Schrödinger equation

\section{Introduction}

The time-dependent Schrödinger equation is a fundamental equation in quantum mechanics for studying the dynamics and evolution of wave packets over time. Several years ago, the classical Schrödinger equation has been generalized to a fractional partial differential equation that takes into account the Riesz space-fractional derivative instead of the conventional Laplacian [1,2]. Apart from quantum mechanics, there are many other equations occurring in science that have been reconsidered in terms of fractional derivatives such as the diffusion-wave equation [3-6], the Langevin equation [7] or the radiative transport equation [8]. Analytical solutions to fractional differential equations are, in general, not available in terms of elementary functions. As an example, the fundamental solution to the Schrödinger equation for a free particle can only be written in closed form under consideration of the Fox $H$-function [9]. Very recently, the space-fractional Schrödinger equation has been employed for studying the propagation dynamics of wave packets in the presence of the harmonic potential [10] as well as of the free particle [11]. In addition, the fractional Schrödinger equation subject to a periodic $\mathcal{P} \mathcal{T}$-symmetric potential has been used to investigate the conical diffraction of a light beam [12]. An optical realization of the space-fractional Schrödinger equation, based on transverse light dynamics in aspherical optical cavities, has been recently proposed in the study [13]. Besides the free particle and the harmonic potential, the case of a linear potential is also a fundamental problem in quantum mechanics that can be treated and solved analytically [14,15]. In this context, we refer to the paper [16] dealing with the nonlinear Schrödinger equation in the presence of uniform acceleration. 
We additionally note on the time-dependent Schrödinger equation with a nonlocal term that has been analyzed in the publication [17].

In this article, we consider the time-dependent Schrödinger equation with the Riesz space-fractional derivative of order $0<\alpha \leq 2$ in the presence of the linear potential. In the one-dimensional (1D) case, the wave function in momentum space is derived for an arbitrary fractional order $\alpha$ in closed form, which allows the determination of other important quantities such as the mean square displacement (MSD). For the case of $\alpha=1$, we provide some explicit analytical solutions useable for studying the time evolution of wave packets that undergo both spreading and splitting. We additionally address the space-fractional Schrödinger equation in two spatial dimensions. The obtained equations are illustrated in different ways and partly verified by comparisons with a recently proposed numerical approach.

\section{1D Space-Fractional Schrödinger Equation}

The 1D space-fractional Schrödinger equation in the presence of the linear potential $V(x)=\beta x$ is given by

$$
i \frac{\partial \psi(x, t)}{\partial t}=\frac{1}{2}(-\Delta)^{\frac{\alpha}{2}} \psi(x, t)+\beta x \psi(x, t), \quad x \in \mathbb{R}, \quad t>0
$$

where $\psi(x, t)$ is the wave function, $0<\alpha \leq 2$ is the order of the Riesz space-fractional derivative and $\beta \in \mathbb{R}$. The nonlocal operator occurring in Equation (1) is defined in the sense of [18]

$$
\mathcal{F}\left\{(-\Delta)^{\frac{\alpha}{2}} f(x)\right\}(\omega)=|\omega|^{\alpha} F(\omega)
$$

with $F(\omega)=\int_{-\infty}^{\infty} f(x) \exp (-i \omega x) d x$ being the Fourier transform of $f(x)$. Alternatively, it can also be defined directly in real space in terms of the left- and right-sided Riemann-Liouville derivatives [19]. In this section, Equation (1) is considered under the initial and boundary conditions

$$
\psi\left(x, 0^{+}\right)=\psi_{0}(x), \quad \lim _{|x| \rightarrow \infty} \psi(x, t)=0
$$

where $\psi_{0}(x)$ is the initial wave packet that is normalized according to $\int_{-\infty}^{\infty}\left|\psi_{0}(x)\right|^{2} d x=1$. The corresponding Schrödinger equation in momentum space is given by

$$
i \frac{\partial \psi(\omega, t)}{\partial t}=\frac{1}{2}|\omega|^{\alpha} \psi(\omega, t)+i \beta \frac{\partial \psi(\omega, t)}{\partial \omega}, \quad \omega \in \mathbb{R}, \quad t>0
$$

where $\psi\left(\omega, 0^{+}\right)=\psi_{0}(\omega)$. In order to solve this equation, we introduce a wave function of the form $\psi(\omega, t)=e^{i \varphi(\omega)} u(\omega+\beta t)$, where $\varphi$ and $u$ are unknown. Inserting this function into Equation (4) results in

$$
i \beta e^{i \varphi} u^{\prime}(\omega+\beta t)=\frac{1}{2}|\omega|^{\alpha} e^{i \varphi} u(\omega+\beta t)+i \beta e^{i \varphi} u^{\prime}(\omega+\beta t)-\beta e^{i \varphi} u(\omega+\beta t) \frac{d \varphi}{d \omega}
$$

where $u^{\prime}$ denotes the first derivative of the unknown function $u$. Comparing both sides of Equation (5) leads to the equation

$$
\frac{d \varphi(\omega)}{d \omega}=\frac{|\omega|^{\alpha}}{2 \beta}
$$

which is solved by the function

$$
\varphi(\omega)=\frac{\omega|\omega|^{\alpha}}{2 \beta(1+\alpha)}+C, \quad \omega \in \mathbb{R}
$$


with $C$ being an arbitrary constant. The unknown quantity $u$ can be found by employing the initial condition according to

$$
\psi(\omega, 0)=\psi_{0}(\omega)=e^{i \varphi(\omega)} u(\omega) \quad \Rightarrow \quad u(\omega)=\psi_{0}(\omega) e^{-i \varphi(\omega)}
$$

Therefore, the general solution of the Schrödinger Equation (4) in momentum space is found to be

$$
\psi(\omega, t)=\psi_{0}(\omega+\beta t) \exp \left(i \frac{\omega|\omega|^{\alpha}-(\omega+\beta t)|\omega+\beta t|^{\alpha}}{2 \beta(1+\alpha)}\right)
$$

for all $0<\alpha \leq 2$. It can be seen that $|\psi(\omega, t)|^{2}=\left|\psi_{0}(\omega+\beta t)\right|^{2}$, which is a measure for the probability density in momentum space, is independent of the fractional order. The wave function in real space is formally given by

$$
\psi(x, t)=\frac{1}{2 \pi} \int_{-\infty}^{\infty} \psi(\omega, t) \exp (i \omega x) d \omega
$$

In general, this integral must be carried out numerically using one of the established quadrature rules. For the classical case of $\alpha=2$, the wave function can also be given in terms of the convolution

$$
\psi(x, t)=\frac{e^{-i \beta x t} e^{-i \beta^{2} t^{3} / 6}}{\sqrt{2 \pi i t}} \int_{-\infty}^{\infty} \psi_{0}(y) \exp \left(i \frac{\left(x-y+\beta t^{2} / 2\right)^{2}}{2 t}\right) d y
$$

The probability density function (pdf) associated with the position of the particle is defined as $\rho(x, t)=|\psi(x, t)|^{2}$. Furthermore, in view of experimental activities, the MSD is known to be an important quantity that can be computed by means of the pdf as $\left\langle x^{2}(t)\right\rangle=\int_{-\infty}^{\infty} x^{2} \rho(x, t) d x$. In our case, when $\rho(x, t)$ is not given, the MSD can be determined from the wave function in momentum space according to

$$
\left\langle x^{2}(t)\right\rangle=\frac{1}{2 \pi} \int_{-\infty}^{\infty}\left|\frac{\partial \psi(\omega, t)}{\partial \omega}\right|^{2} d \omega
$$

Using Equation (9), one obtains the MSD for an arbitrary initial stage as

$$
\left\langle x^{2}(t)\right\rangle=\frac{1}{2 \pi} \int_{-\infty}^{\infty}\left|\frac{d \psi_{0}(\omega)}{d \omega}+i \psi_{0}(\omega) \frac{|\omega-\beta t|^{\alpha}-|\omega|^{\alpha}}{2 \beta}\right|^{2} d \omega
$$

In the following, we also want to address the case of the free particle which is obtained for $\beta=0$. The corresponding solution in momentum space Equation (9) reduces to

$$
\psi(\omega, t)=\psi_{0}(\omega) \exp \left(-i t|\omega|^{\alpha} / 2\right), \quad 0<\alpha \leq 2
$$

However, also in this case, the wave function in real space is not available in elementary form. Instead, we have

$$
\psi(x, t)=\int_{-\infty}^{\infty} G(x-y, t) \psi_{0}(y) d y
$$

with $G(x, t)$ being Green's function, which is given for $1<\alpha \leq 2$ by

$$
G(x, t)=\frac{1}{\sqrt{\pi}|x|} H_{1,2}^{1,1}\left[\frac{2}{i t} \frac{|x|^{\alpha}}{2^{\alpha}} \mid \begin{array}{l}
(1,1) \\
(1 / 2, \alpha / 2),(1, \alpha / 2)
\end{array}\right]
$$

The Fox $H$-function occurring above is defined in terms of the Mellin-Barnes integral [9]

$$
H_{p, q}^{m, n}\left[z \mid \begin{array}{c}
\left(a_{p}, A_{p}\right) \\
\left(b_{q}, B_{q}\right)
\end{array}\right]=\frac{1}{2 \pi i} \int_{L} \frac{\prod_{j=1}^{m} \Gamma\left(b_{j}+B_{j} s\right) \prod_{j=1}^{n} \Gamma\left(1-a_{j}-A_{j} s\right)}{\prod_{j=n+1}^{p} \Gamma\left(a_{j}+A_{j} s\right) \prod_{j=m+1}^{q} \Gamma\left(1-b_{j}-B_{j} s\right)} z^{-s} \mathrm{~d} s
$$


where $\Gamma(s)$ is the Gamma function and $L$ denotes an appropriate integration path in the complex plane. The fundamental solution Equation (16) can be evaluated by means of the series

$$
G(x, t)=\frac{(-2 i / t)^{1 / \alpha}}{\alpha \pi} \sum_{k=0}^{\infty} \frac{\left(-(-2 i / t)^{2 / \alpha} x^{2}\right)^{k}}{(2 k) !} \Gamma\left(\frac{2 k+1}{\alpha}\right)
$$

where $G(0, t)=(-2 i / t)^{1 / \alpha} \Gamma(1 / \alpha) /(\alpha \pi)$. The corresponding MSD for $\beta=0$ becomes

$$
\left\langle x^{2}(t)\right\rangle=\left.\left.\frac{1}{2 \pi} \int_{-\infty}^{\infty}\left|\frac{d \psi_{0}(\omega)}{d \omega}-i \psi_{0}(\omega) \frac{\alpha t}{2} \operatorname{sign}(\omega)\right| \omega\right|^{\alpha-1}\right|^{2} d \omega
$$

where $\operatorname{sign}(x)$ is the sign function. It can be verified that the MSD Equation (19) as function of time describes for all fractional orders a parabola. As an example, for the commonly applied Gaussian wave packet

$$
\psi_{0}(x)=\frac{1}{\sqrt{\sigma \sqrt{\pi}}} \exp \left(-\frac{x^{2}}{2 \sigma^{2}}\right)
$$

one obtains in the free particle case the MSD

$$
\left\langle x^{2}(t)\right\rangle=\frac{(\alpha \sigma t)^{2}}{(2 \sigma)^{2 \alpha}} \frac{\Gamma(2 \alpha-1)}{\Gamma(\alpha)}+\frac{\sigma^{2}}{2}
$$

which exists for all $1 / 2<\alpha \leq 2$. Figure 1 displays the MSD Equation (21) for different values of the fractional order.

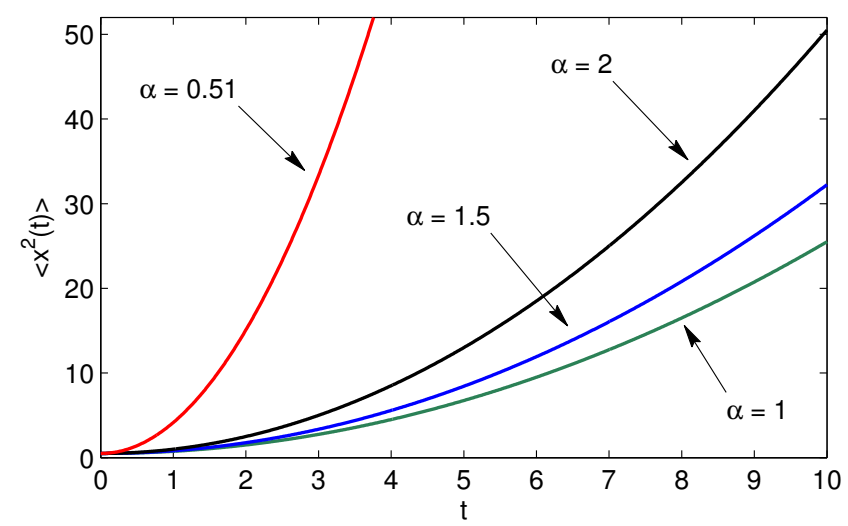

Figure 1. Illustration of the mean square displacement (MSD) Equation (21) for different values of the fractional order $\alpha$ under consideration of the Gaussian initial wave packet Equation (20) with $\sigma=1$.

Explicit Solutions for the Square Root of the Laplacian $(-\Delta)$

In this subsection, we provide some explicit analytical solutions of Equation (1) for the order $\alpha=1$. In this case, the fractional Laplacian can be represented as [20]

$$
(-\Delta)^{\frac{1}{2}} f(x)=\mathcal{F}^{-1}\{|\omega| F(\omega)\}(x)=\frac{d}{d x} \mathcal{H}\{f(x)\}
$$

where $\mathcal{H}$ is the Hilbert transform that is defined by

$$
\mathcal{H}\{f(x)\}=\frac{1}{\pi} \mathcal{P} \int_{-\infty}^{\infty} \frac{f(y)}{x-y} d y
$$

and $\mathcal{P}$ denotes the Cauchy principal value. It should be noted that, in recent time, the case of $\alpha=1$ has attracted attention in the context of the propagation dynamics of wave packets under the use of 
the space-fractional Schrödinger equation $[10,11,13]$. In this study, we investigate the time evolution of the Cauchy initial wave packet

$$
\psi_{0}(x)=\sqrt{\frac{2 \varepsilon}{\pi}} \frac{\varepsilon}{x^{2}+\varepsilon^{2}}
$$

where $\varepsilon>0$ is a measure for the beam width. We afore have to note that the time evolution of the Gaussian wave packet and the Cauchy wave packet shows a similar behavior. However, in the latter case, we are able to derive simple analytical solutions that are easy to implement and reproduce. Our starting point is the evaluation of the Fourier integral Equation (10) under consideration of the wave function Equation (9). For this, we also need the Cauchy wave packet Equation (24) in momentum space, that is $\psi_{0}(\omega)=\sqrt{2 \pi \varepsilon} \exp (-\varepsilon|\omega|)$. Splitting the integrand into three sections and assuming that $\beta \geq 0$ enables the derivation of the following closed-form expression

$$
\begin{aligned}
\psi(x, t)= & \sqrt{\frac{\varepsilon}{2 \pi}} e^{-i \beta t^{2} / 4}\left[\frac{e^{-\beta \varepsilon t}}{\varepsilon-i(x-t / 2)}+\frac{e^{-i \beta x t}}{\varepsilon+i(x+t / 2)}\right]+\frac{1}{2} \sqrt{\frac{\beta \varepsilon}{i}} e^{-\beta t(\varepsilon+i t / 4)} \\
& \times e^{i \beta(x-t / 2+i \varepsilon)^{2} / 2}\left[\operatorname{erf} \sqrt{\frac{i \beta}{2}}(x+t / 2+i \varepsilon)-\operatorname{erf} \sqrt{\frac{i \beta}{2}}(x-t / 2+i \varepsilon)\right]
\end{aligned}
$$

where $\operatorname{erf}(z)$ is the error function. Note that the case $\beta<0$ can be treated in the same manner. The associated MSD belonging to this process can be obtained from Equation (13) and summarized as

$$
\left\langle x^{2}(t)\right\rangle=\frac{t^{2}}{4}-\frac{1+e^{-2 \beta \varepsilon t}}{4 \beta \varepsilon} t+\frac{1+4 \beta^{2} \varepsilon^{4}-e^{-2 \beta \varepsilon t}}{(2 \beta \varepsilon)^{2}}
$$

In this context, we note on the limits

$$
\lim _{\beta \rightarrow 0}\left\langle x^{2}(t)\right\rangle=\lim _{\beta \rightarrow \infty}\left\langle x^{2}(t)\right\rangle=\frac{t^{2}}{4}+\varepsilon^{2}
$$

In the case of the free particle, there is the possibility to provide the general solution for an arbitrary initial stage. In doing so, we need the inverse Fourier transform of the impulse response $G(\omega, t)=\exp (-i t|\omega| / 2)$, which can be written as

$$
G(x, t)=\frac{1}{2 \pi} \int_{-\infty}^{\infty} \exp (-i t|\omega| / 2) \exp (i \omega x) d \omega=\frac{t}{2} \delta\left(x^{2}-t^{2} / 4\right)+\frac{i}{\pi} \frac{2 t}{4 x^{2}-t^{2}}
$$

Inserting this finding into Equation (15) leads to the general solution of the Schrödinger equation

$$
\psi(x, t)=\frac{\psi_{0}(x-t / 2)+\psi_{0}(x+t / 2)}{2}+\frac{i t}{2 \pi} \mathcal{P} \int_{-\infty}^{\infty} \frac{\psi_{0}(y)}{(x-y)^{2}-t^{2} / 4} d y
$$

In the actual case of the Cauchy initial wave packet Equation (24), we find the time evolution

$$
\psi(x, t)=\sqrt{\frac{\varepsilon}{2 \pi}} \frac{2 \varepsilon+i t}{x^{2}+(\varepsilon+i t / 2)^{2}}
$$

which can be confirmed via Equation (25) by setting $\beta=0$. The resulting pdf belonging to this process is

$$
\rho(x, t)=\frac{2 \varepsilon}{\pi} \frac{\varepsilon^{2}+t^{2} / 4}{\left(x^{2}+\varepsilon^{2}-t^{2} / 4\right)^{2}+(\varepsilon t)^{2}}
$$

In addition, the cumulative distribution function $F(x, t)=\int_{-\infty}^{x} \rho(y, t) d y$ is found to be

$$
F(x, t)=\frac{1}{2}+\frac{t^{2}+4 \varepsilon^{2}}{2 \pi t} \operatorname{Im}\left(\frac{\arctan [x /(\varepsilon-i t / 2)]}{\varepsilon-i t / 2}\right)
$$


Within the classical quantum mechanics, it is known that a pdf belonging to the free particle solution typically spreads out but it does not split. However, as has been very recently shown, the pdf belonging to the space-fractional Schrödinger equation additionally undergoes splitting [11]. For the Cauchy wave packet, there is the possibility to provide some more details concerning this feature. Taking the first derivative of the pdf Equation (31) with respect to the space and setting $\rho_{x}(x, t)=0$, yields three critical positions, namely $x=0$ or $x= \pm \sqrt{t^{2} / 4-\varepsilon^{2}}$. From the behavior of $\rho(x, t)$, it follows that $x=0$ is an absolute maximum as long as $0 \leq t \leq 2 \varepsilon$. On the other side, for time values $t>2 \varepsilon$, the position $x=0$ becomes a relative minimum. At the same time, we obtain two absolute maxima located at $x= \pm \sqrt{t^{2} / 4-\varepsilon^{2}}$. Consequently, the probability density spreads out for $t>0$, and additionally, starts to split at $t=2 \varepsilon$. For illustration purposes, Figure 2 shows the time evolution of a Cauchy initial wave packet according to Equation (31).

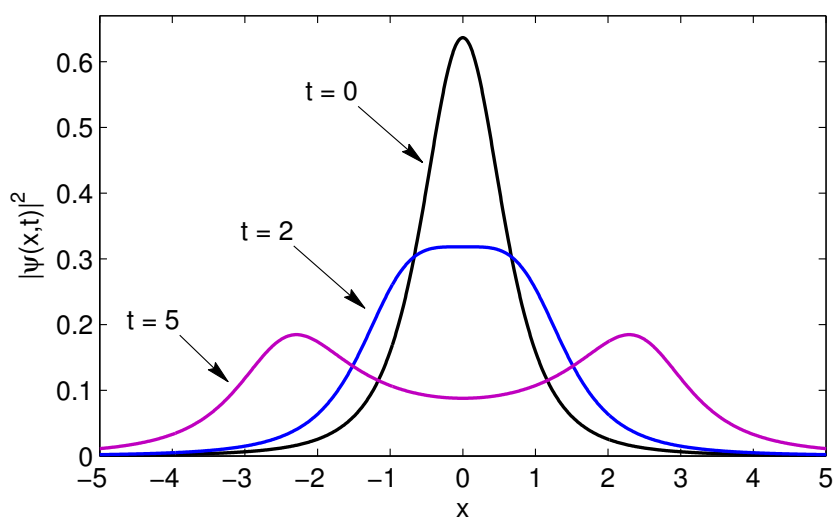

Figure 2. Time evolution of the Cauchy initial wave packet Equation (24) for the parameter $\varepsilon=1$.

A similar behavior can be observed in the case of the Gaussian initial wave packet Equation (20), where the critical time regarding the splitting process must be determined numerically from the wave function

$$
\psi(x, t)=\frac{\psi_{0}(x-t / 2)}{2}\left[1+i \operatorname{erfi}\left(\frac{x-t / 2}{\sqrt{2} \sigma}\right)\right]+\frac{\psi_{0}(x+t / 2)}{2}\left[1-i \operatorname{erfi}\left(\frac{x+t / 2}{\sqrt{2} \sigma}\right)\right]
$$

where erfi $(x)$ is the imaginary error function and $\psi_{0}(x)$ is given by Equation (20). Figure 3 displays the same situation as shown in Figure 2 for the the Gaussian initial wave packet Equation (20).

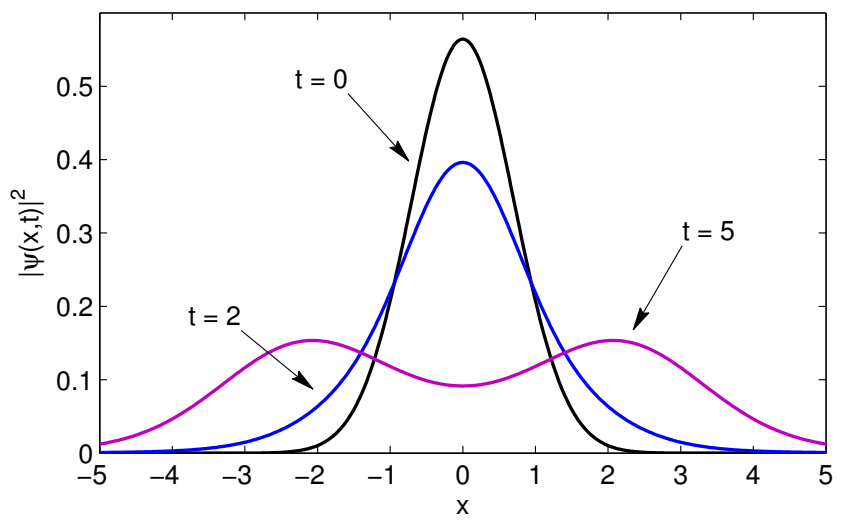

Figure 3. Time evolution of the Gaussian initial wave packet Equation (20) for the parameter $\sigma=1$.

Concerning an experimental verification/realization of the derived theoretical results we refer to the recent publication [11], which proposes an optical system composed of two convex lenses and a 
phase mask. We furthermore note on the free particle solution to the classical Schrödinger equation. In the case of the Cauchy wave packet Equation (24), we have

$$
\psi(x, t)=\frac{1}{2} \sqrt{\frac{\varepsilon}{i t}}\left[w\left(\frac{i \varepsilon+x}{\sqrt{2 i t}}\right)+w\left(\frac{i \varepsilon-x}{\sqrt{2 i t}}\right)\right]
$$

where $w(z)=\exp \left(-z^{2}\right) \operatorname{erfc}(-i z)$ is the Faddeeva function. The corresponding MSD belonging to this process is $\left\langle x^{2}(t)\right\rangle=t^{2} /\left(2 \varepsilon^{2}\right)+\varepsilon^{2}$. Figure 4 displays the time evolution of the wave function Equation (34) for the same time values considered in Figure 2. It can can be seen that the probability density indeed spreads out but it does not split.

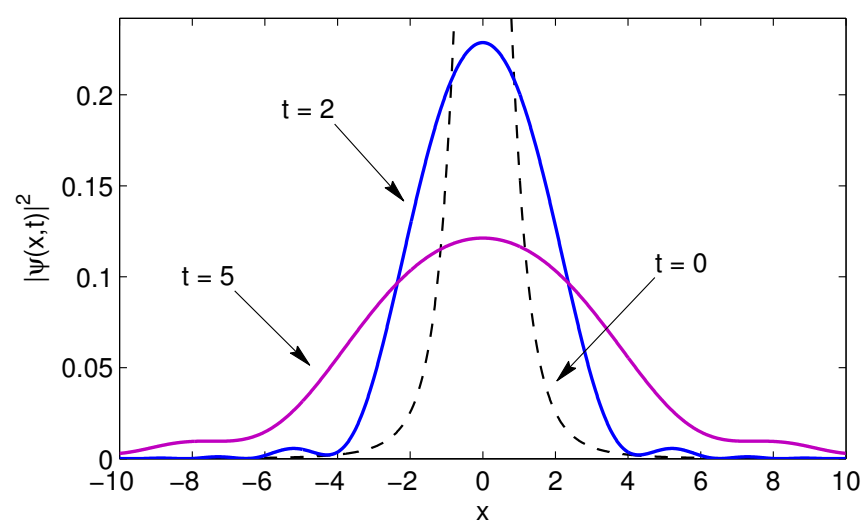

Figure 4. Time evolution of the Cauchy initial wave packet Equation (24) for $\varepsilon=1$ within the classical quantum mechanics.

\section{2D Space-Fractional Schrödinger Equation}

The 2D space-fractional Schrödinger equation in the presence of the potential $V(\boldsymbol{\rho})=\mathbf{F} \cdot \boldsymbol{\rho}$ is given by

$$
i \frac{\partial \psi(\rho, t)}{\partial t}=\frac{1}{2}(-\Delta)^{\frac{\alpha}{2}} \psi(\rho, t)+V(\boldsymbol{\rho}) \psi(\boldsymbol{\rho}, t), \quad \boldsymbol{\rho} \in \mathbb{R}^{2}, \quad t>0
$$

where $\mathbf{F}=\left(F_{1}, F_{2}\right)$ and $F=|\mathbf{F}|$. Again, the 2D fractional Laplacian is most convenient defined by

$$
\mathcal{F}\left\{(-\Delta)^{\frac{\alpha}{2}} f(\boldsymbol{\rho})\right\}(\mathbf{q})=|\mathbf{q}|^{\alpha} F(\mathbf{q})
$$

where $|\mathbf{q}|=q$ denotes the length of the wave vector $\mathbf{q}=\left(q_{1}, q_{2}\right)$ that belongs to the Fourier transform $F(\mathbf{q})=\int f(\boldsymbol{\rho}) \exp (-i \mathbf{q} \cdot \boldsymbol{\rho}) d \boldsymbol{\rho}$. In addition, for $\alpha=1$, the 2D fractional Laplacian can be represented as

$$
(-\Delta)^{\frac{1}{2}} f(\rho)=\nabla \cdot \mathcal{R}\{f(\rho)\}
$$

where $\mathcal{R}$ denotes the Riesz transform [21], that maps a scalar function $f(\rho)$ into a vector field according to

$$
\mathcal{R}\{f(\boldsymbol{\rho})\}=\frac{1}{2 \pi} \int \frac{f(\mathbf{s})(\boldsymbol{\rho}-\mathbf{s})}{|\boldsymbol{\rho}-\mathbf{s}|^{3}} d \mathbf{s}
$$

We note that relation Equation (37) is a generalization of the 1D differential operator Equation (22). Concerning the initial and boundary conditions in 2D, we have

$$
\psi\left(\boldsymbol{\rho}, 0^{+}\right)=\psi_{0}(\boldsymbol{\rho}), \quad \lim _{\rho \rightarrow \infty} \psi(\boldsymbol{\rho}, t)=0
$$


where $\rho=|\rho|$. The Schrödinger Equation (35) in momentum space becomes

$$
\frac{\partial \psi(\mathbf{q}, t)}{\partial t}=\frac{q^{\alpha}}{2 i} \psi(\mathbf{q}, t)+\mathbf{F} \cdot \nabla \psi(\mathbf{q}, t), \quad \mathbf{q} \in \mathbb{R}^{2}, \quad t>0
$$

where the Del operator has to be applied with regard to the Fourier variables. Similar as in 1D, we seek a wave function of the form $\psi(\mathbf{q}, t)=\exp [i \varphi(\mathbf{q})] u(\mathbf{q}+\mathbf{F} t)$. Inserting this ansatz into Equation (40), leads to the first order partial differential equation

$$
F_{1} \frac{\partial \varphi(\mathbf{q})}{\partial q_{1}}+F_{2} \frac{\partial \varphi(\mathbf{q})}{\partial q_{2}}=\frac{q^{\alpha}}{2}
$$

In contrast to the 1D case, we are not able to solve this equation for all fractional orders in elementary form. However, for the relevant case of $\alpha=1$, an exact solution can be derived and summarized as

$$
\varphi(\mathbf{q})=\frac{q}{4} \frac{F_{1} q_{1}+F_{2} q_{2}}{F_{1}^{2}+F_{2}^{2}}+\frac{1}{4} \frac{\left(F_{1} q_{2}-F_{2} q_{1}\right)^{2}}{\left(F_{1}^{2}+F_{2}^{2}\right)^{3 / 2}} \ln \left(2 q+2 \frac{F_{1} q_{1}+F_{2} q_{2}}{\sqrt{F_{1}^{2}+F_{2}^{2}}}\right)
$$

The solution belonging to the classical case with $\alpha=2$ is less complicated and given by $\varphi(\mathbf{q})=$ $q_{1}^{3} /\left(3 F_{1}\right)+q_{2}^{3} /\left(3 F_{2}\right)$. Again, the unknown function $u$ can be recovered under consideration of the initial condition such as

$$
\psi(\mathbf{q}, 0)=\psi_{0}(\mathbf{q})=\exp [i \varphi(\mathbf{q})] u(\mathbf{q}) \quad \Rightarrow \quad u(\mathbf{q})=\psi_{0}(\mathbf{q}) \exp [-i \varphi(\mathbf{q})]
$$

Therefore, we obtain the wave function in momentum space according to

$$
\psi(\mathbf{q}, t)=\psi_{0}(\mathbf{q}+\mathbf{F} t) \exp [i(\varphi(\mathbf{q})-\varphi(\mathbf{q}+\mathbf{F} t))]
$$

In the case of the free particle with $\mathbf{F}=\mathbf{0}$, the solution in momentum space can be given for all orders as

$$
\psi(\mathbf{q}, t)=\psi_{0}(\mathbf{q}) \exp \left(-i t q^{\alpha} / 2\right), \quad 0<\alpha \leq 2
$$

Moreover, if the initial wave packet exhibits rotational symmetry which implies that $\psi_{0}(\mathbf{q})=$ $\psi_{0}(q)$, the solution in real space can be evaluated by means of the inverse Hankel transform

$$
\psi(\rho, t)=\frac{1}{2 \pi} \int_{0}^{\infty} \psi_{0}(q) \exp \left(-i t q^{\alpha} / 2\right) J_{0}(q \rho) q d q, \quad 0<\alpha \leq 2
$$

with $J_{0}(x)$ being the zero order Bessel function. For $\psi_{0}(q)=1$ and $1<\alpha \leq 2$, Equation (46) yields the 2D Green's function

$$
G(\rho, t)=\frac{1}{\pi \rho^{2}} H_{1,2}^{1,1}\left[\begin{array}{l|l}
\frac{2}{i t} \frac{\rho^{\alpha}}{2^{\alpha}} & \left.\begin{array}{l}
(1,1) \\
(1, \alpha / 2),(1, \alpha / 2)
\end{array}\right]
\end{array}\right.
$$

which can be evaluated by means of the series

$$
G(\rho, t)=\frac{(-2 i / t)^{2 / \alpha}}{2 \alpha \pi} \sum_{k=0}^{\infty} \frac{\left(-(-2 i / t)^{2 / \alpha} \rho^{2} / 4\right)^{k}}{(k !)^{2}} \Gamma\left(\frac{2 k+2}{\alpha}\right)
$$

where $G(0, t)=(-2 i / t)^{2 / \alpha} \Gamma(2 / \alpha) /(2 \alpha \pi)$. In the case of $\alpha=2$, this relation coincides with the known result $G(\rho, t)=\exp \left(i \rho^{2} /(2 t)\right) /(2 \pi i t)$. On the other side, Green's function for $\alpha=1$ and $\rho \neq t / 2$ is found to be

$$
G(\rho, t)=\frac{2 i}{\pi} \frac{\partial}{\partial t} \frac{1}{\sqrt{4 \rho^{2}-t^{2}}}=\frac{2}{\pi} \frac{i t}{\left(4 \rho^{2}-t^{2}\right)^{3 / 2}}
$$


Based on this relation, we obtain the wave function

$$
\psi(\boldsymbol{\rho}, t)=\frac{2 i}{\pi} \frac{\partial}{\partial t} \int \frac{\psi_{0}(\boldsymbol{\rho}-\mathbf{s})}{\sqrt{4 s^{2}-t^{2}}} d \mathbf{s}
$$

which is the general solution of Equation (35) for $\alpha=1$ and $\mathbf{F}=\mathbf{0}$. In the following, we investigate the time evolution of the rotationally symmetric Cauchy wave packet

$$
\psi_{0}(\rho)=\sqrt{\frac{2}{\pi}} \frac{\varepsilon^{2}}{\left(\rho^{2}+\varepsilon^{2}\right)^{3 / 2}}
$$

which is normalized according to $\int\left|\psi_{0}(\rho)\right|^{2} d \rho=1$. Similar as in $1 \mathrm{D}$, we consider the case of $\alpha=1$ in detail. The desired wave function can be obtained either via the convolution Equation (50) or by means of the inverse Hankel transform Equation (46). In the latter case, we need the initial wave packet Equation (51) in momentum space, that is $\psi_{0}(q)=2 \varepsilon \sqrt{2 \pi} \exp (-\varepsilon q)$. The required inverse Hankel transform can be taken exactly and summarized as

$$
\psi(\rho, t)=\sqrt{\frac{2}{\pi}} \frac{\varepsilon(\varepsilon+i t / 2)}{\left[\rho^{2}+(\varepsilon+i t / 2)^{2}\right]^{3 / 2}}
$$

The associated probability density to this wave function is given by

$$
|\psi(\rho, t)|^{2}=\frac{2 \varepsilon^{2}}{\pi} \frac{\varepsilon^{2}+t^{2} / 4}{\left[\left(\rho^{2}+\varepsilon^{2}-t^{2} / 4\right)^{2}+(\varepsilon t)^{2}\right]^{3 / 2}}
$$

In accordance with the 1D case, the probability density in 2D also undergoes spreading and splitting. Remarkable, the probability density Equation (53) starts to split at $t=2 \varepsilon$, which is exactly the same time value as for the $1 \mathrm{D}$ pendant Equation (31). Figure 5 displays the time evolution of a rotationally symmetric Cauchy initial wave packet for different time values.

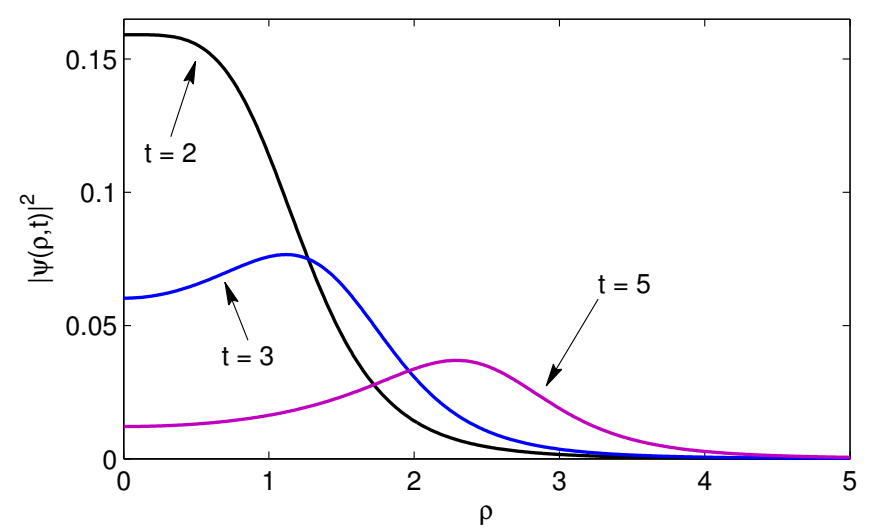

Figure 5. Time evolution of the rotationally symmetric Cauchy initial wave packet Equation (51) for the parameter $\varepsilon=1$.

We note that the solution for the rotationally symmetric Gaussian wave packet $\psi_{0}(\rho)=$ $2 \exp \left(-2 \rho^{2} / \sigma^{2}\right) /(\sqrt{\pi} \sigma)$ can be derived in a similar manner. The required Hankel transform for evaluation of the wave function Equation (46) is given by $\psi_{0}(q)=\sqrt{\pi} \sigma \exp \left(-\sigma^{2} q^{2} / 8\right)$.

\section{Numerical Solution of the Fractional Schrödinger Equation}

In this section, we describe a recently proposed matrix approach [22] that has been used for solving numerically the 1D space-fractional diffusion equation. In this context, we additionally refer to the recent publication [23], which takes into account the so-called Adomian decomposition method. 
We adopt the matrix approach in order to solve the space-fractional Schrödinger Equation (1) subject to an arbitrary potential function $V(x)$. Within this framework, we consider the computational domain $|x| \leq L / 2$ together with the uniform grid $x_{j}=-L / 2+j h$ for $j=0, \ldots, N$ as well as the node spacing $h=L / N$. Furthermore, the fractional Laplacian has to be restricted to a bounded domain. If $0<\alpha<1$, we have the representation

$$
(-\Delta)^{\frac{\alpha}{2}} f(x)=\frac{1}{2 \cos (a \pi / 2) \Gamma(1-a)} \frac{d}{d x} \int_{-L / 2}^{L / 2} \frac{\operatorname{sign}(x-y) f(y)}{|x-y|^{\alpha}} d y
$$

whereas for $1<\alpha<2$ it is given by

$$
(-\Delta)^{\frac{\alpha}{2}} f(x)=\frac{1}{2 \cos (a \pi / 2) \Gamma(2-a)} \frac{d^{2}}{d x^{2}} \int_{-L / 2}^{L / 2} \frac{f(y)}{|x-y|^{\alpha-1}} d y
$$

In particular, the square root of the negative Laplacian on a bounded domain becomes

$$
(-\Delta)^{\frac{1}{2}} f(x)=\frac{1}{\pi} \frac{d}{d x} \int_{-L / 2}^{L / 2} \frac{f(y)}{x-y} d y
$$

For our applications, the quantity $L$ has to be chosen relatively large in order to obtain a reasonable model for the infinite domain. On the other side, the matrix approach is designed for solving the fractional Schrödinger on a bounded domain, where analytical solutions are typically not available. The key point here is the approximation of the fractional Laplacian by means of the fractional centered difference [22]

$$
(-\Delta)^{\frac{\alpha}{2}} f\left(x_{j}\right) \approx \frac{1}{h^{\alpha}} \sum_{k=1}^{N-1} g_{|j-k|} f\left(x_{k}\right)
$$

which can be applied for all $0<\alpha \leq 2$. The corresponding weights are given by [22]

$$
g_{k}=\frac{(-1)^{k} \Gamma(1+\alpha)}{\Gamma(1+k+\alpha / 2) \Gamma(1-k+\alpha / 2)}
$$

We note that within the definition Equation (57), we have taken into account the boundary data $f\left(x_{0}\right)=f\left(x_{N}\right)=0$. The weights given in Equation (58) can be computed efficiently by means of the recursion [22]

$$
g_{k+1}=\frac{2 k-\alpha}{2 k+\alpha+2} g_{k}
$$

Applying the fractional centered difference scheme Equation (57) to the Schrödinger Equation (1) leads to the following system of ordinary differential equations

$$
i \frac{d \psi_{j}(t)}{d t}=\frac{1}{2 h^{\alpha}} \sum_{k=1}^{N-1} g_{|j-k|} \psi_{k}(t)+V_{j} \psi_{j}(t)
$$

where $j=1, \ldots, N-1, \psi_{j}(t)=\psi\left(x_{j}, t\right)$ and $V_{j}=V\left(x_{j}\right)$. The system Equation (60) in matrix notation becomes

$$
\frac{d \boldsymbol{\Phi}(t)}{d t}=-i M \boldsymbol{\Phi}(t)
$$

where $\boldsymbol{\Phi}(t)=\left(\psi_{1}(t), \psi_{2}(t), \ldots, \psi_{N-1}(t)\right)$. The associated solution can be directly given in terms of the matrix exponential according to

$$
\boldsymbol{\Phi}(t)=\exp (-i M t) \boldsymbol{\Phi}_{0}
$$


where $\boldsymbol{\Phi}_{0}=\left(\psi_{0}\left(x_{1}\right), \psi_{0}\left(x_{2}\right), \ldots, \psi_{0}\left(x_{N-1}\right)\right)$ is a column vector that contains the sampled initial wave packet $\psi_{0}(x)$. If MATLAB is used, the matrix exponential can be evaluated via the function expm. For illustration purposes, the corresponding matrix $M$ in the case of $N=5$ looks like

$$
M=\frac{1}{2 h^{\alpha}}\left(\begin{array}{llll}
g_{0} & g_{1} & g_{2} & g_{3} \\
g_{1} & g_{0} & g_{1} & g_{2} \\
g_{2} & g_{1} & g_{0} & g_{1} \\
g_{3} & g_{2} & g_{1} & g_{0}
\end{array}\right)+\left(\begin{array}{cccc}
V_{1} & 0 & 0 & 0 \\
0 & V_{2} & 0 & 0 \\
0 & 0 & V_{3} & 0 \\
0 & 0 & 0 & V_{4}
\end{array}\right)
$$

Now, the structure of the matrix $M$ can be readily continued for an arbitrary $N$. In the following, we use the above described numerical scheme in order to solve the space-fractional Schrödinger Equation (1). We take into account the Cauchy initial wave packet Equation (24) with $\varepsilon=1$ and evaluate the resulting probability density for $\alpha=1$ and $t=5$. The size of the computational domain for approximation of the infinite domain is set to $L=30$. Figure 6 displays the comparison between the analytical solution Equation (31) and the matrix approach Equation (62) for the case of a free particle.

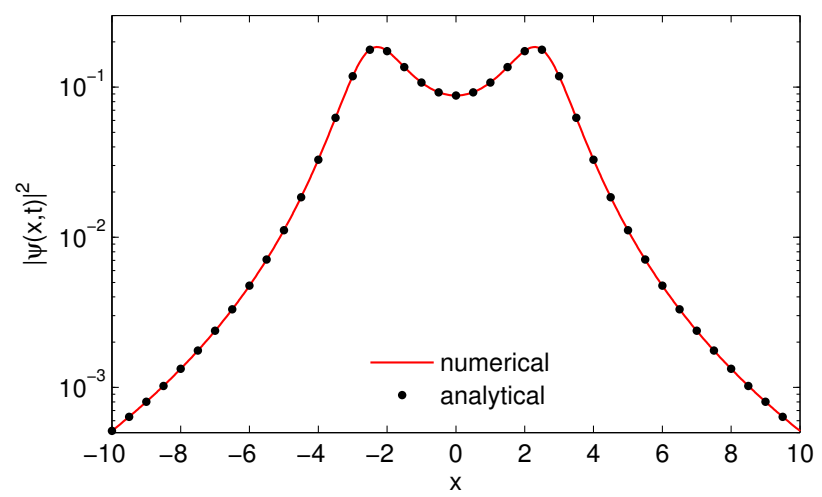

Figure 6. Comparison between the analytical solution Equation (31) and the matrix approach Equation (62) for the case of a free particle.

Next, we consider the case of the linear potential with $\beta=0.5$. The resulting probability density belonging to this process is shown in Figure 7. In contrast to the free particle, the probability density is no longer symmetric and additionally exhibits some oscillations.

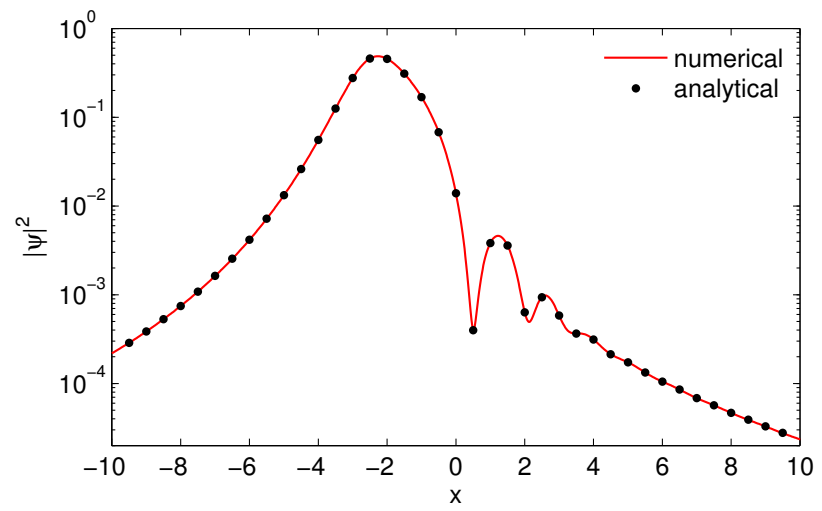

Figure 7. Comparison between the analytical solution Equation (25) and the matrix approach Equation (62) for the case of a liner potential with $\beta=0.5$.

We repeat the last numerical experiment for the case of a stronger accelerated particle with $\beta=4$. The resulting probability density, which is depicted in Figure 8 , is given by a smooth curve 
without oscillations. Furthermore, in contrast to the free particle case, the probability density has not been splitted.

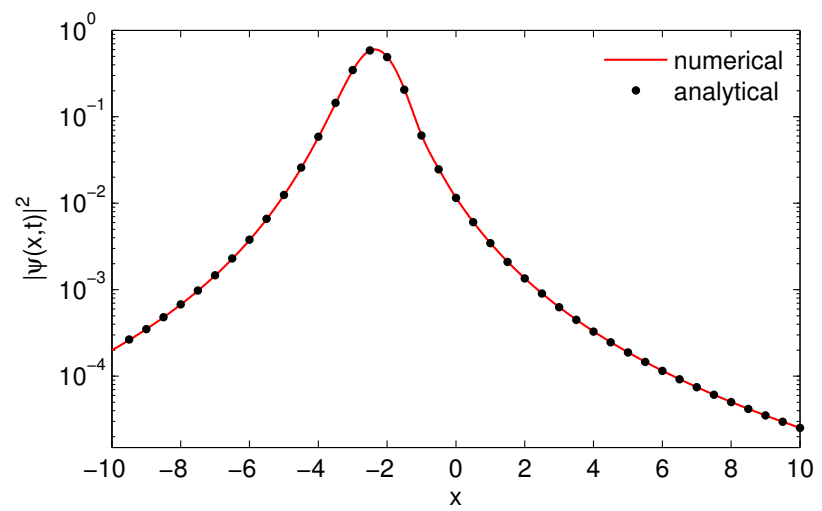

Figure 8. Comparison between the analytical solution Equation (25) and the matrix approach Equation (62) for the case of a liner potential with $\beta=4$.

The numerical experiments outlined above confirm both the correctness of the analytical solutions as well as the good quality of the matrix approach. Besides these comparisons, we additionally have performed numerical experiments for other fractional orders $\alpha$. As a result, we observed in all cases the same good agreement between the analytical solution Equation (10) and the matrix approach Equation (62). It should also be noted that the above matrix approach can be readily extended for solving the space-time fractional Schrödinger equation. More precisely, if we replace the classical time derivative occurring in Equation (1) by the Caputo derivative [24] of order $0<v \leq 1$

$$
D_{t}^{v} f(t)=\frac{1}{\Gamma(1-v)} \int_{0}^{t} \frac{f^{\prime}(\tau)}{(t-\tau)^{v}} d \tau
$$

one obtains the following system of fractional differential equations

$$
D_{t}^{v} \boldsymbol{\Phi}(t)=-i M \boldsymbol{\Phi}(t)
$$

The solution to this system is formally given by $\boldsymbol{\Phi}(t)=E_{v}\left(-i M t^{v}\right) \boldsymbol{\Phi}_{0}$, where $E_{v}(A)=$ $\sum_{n=0}^{\infty} A^{n} / \Gamma(1+v n)$ is the Mittag-Leffler matrix function. It can be computed under consideration of the eigenvalue decomposition $A=U \Lambda U^{-1}$, where $U$ is a matrix containing the eigenvectors of $A$. For $A \in \mathbb{C}^{n \times n}$ with eigenvalues $\Lambda=\operatorname{diag}\left(\lambda_{1}, \lambda_{2}, \ldots, \lambda_{n}\right)$, we have $E_{v}(A)=U E_{v}(\Lambda) U^{-1}$ together with the diagonal matrix

$$
E_{v}(\Lambda)=\left(\begin{array}{cccc}
E_{v}\left(\lambda_{1}\right) & 0 & \cdots & 0 \\
0 & E_{v}\left(\lambda_{2}\right) & \cdots & 0 \\
\vdots & \vdots & \ddots & \vdots \\
0 & 0 & \cdots & E_{v}\left(\lambda_{n}\right)
\end{array}\right)
$$

As a final numerical experiment, we consider the space-time fractional Schrödinger equation

$$
i D_{t}^{\alpha} \psi(x, t)=(-\Delta)^{\frac{\alpha}{2}} \psi(x, t) / 2, \quad x \in \mathbb{R}, \quad t>0
$$

for the joint fractional order $\alpha \in(0,1)$. In this case, the wave function in momentum space can be written as $\psi(\omega, t)=\psi_{0}(\omega) G(\omega, t)$, where $G(\omega, t)=E_{\alpha}\left(-i|\omega|^{\alpha} t^{\alpha} / 2\right)$. The corresponding Green's function in space domain is available in elementary form as

$$
G(x, t)=\frac{1}{2 \pi} \int_{-\infty}^{\infty} E_{\alpha}\left(-i|\omega|^{\alpha} t^{\alpha} / 2\right) \exp (i \omega x) d \omega=\frac{i}{2 \pi} \frac{|x|^{\alpha-1} t^{\alpha} \sin (\alpha \pi / 2)}{|x|^{2 \alpha}+i|x|^{\alpha} t^{\alpha} \cos (\alpha \pi / 2)-t^{2 \alpha} / 4}
$$


We note that this result can be derived in a similar manner as the fundamental solution to the neutral-fractional wave equation [25]. The response to an arbitrary initial stage is given by the convolution

$$
\psi(x, t)=\frac{i t^{\alpha} \sin (\alpha \pi / 2)}{2 \alpha \pi} \int_{0}^{\infty} \frac{\psi_{0}\left(x-u^{1 / \alpha}\right)+\psi_{0}\left(x+u^{1 / \alpha}\right)}{u^{2}+i u t^{\alpha} \cos (\alpha \pi / 2)-t^{2 \alpha} / 4} d u
$$

In most cases, this integral must be evaluated numerically. However, for the Cauchy initial wave packet Equation (24) the integration can be carried out analytically and summarized as

$$
\psi(x, t)=\sqrt{\frac{\varepsilon}{2 \pi}}\left[\frac{(\varepsilon+i x)^{\alpha-1}}{(\varepsilon+i x)^{\alpha}+i t^{\alpha} / 2}+\frac{(\varepsilon-i x)^{\alpha-1}}{(\varepsilon-i x)^{\alpha}+i t^{\alpha} / 2}\right]
$$

In Figure 9 we have evaluated the solution of the space-time fractional Schrödinger equation for two different fractional orders subject to the initial stage Equation (20). The analytical solution Equation (69) is denotes by filled dots, whereas the solid line is the numerical solution obtained by means of the Mittag-Leffler matrix function. In both cases the wave function is evaluated for the time value $t=5$.

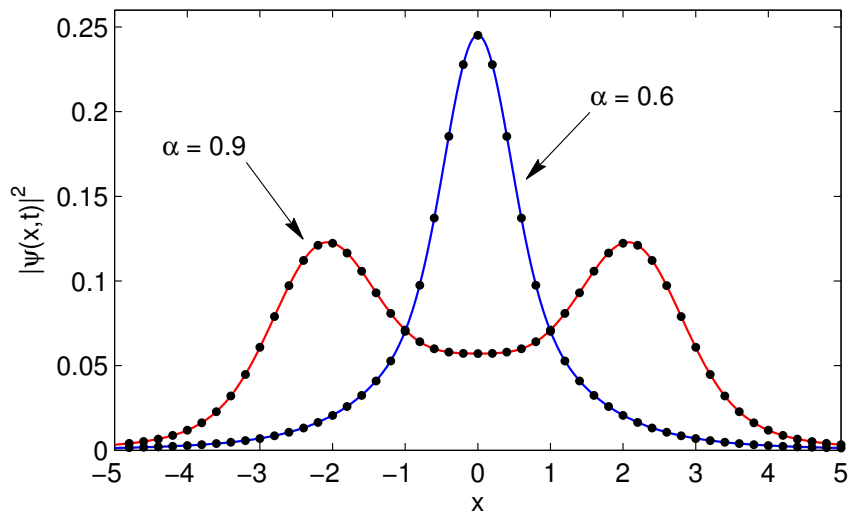

Figure 9. Comparison between the analytical solution Equation (69) and the matrix approach for the case of the Gaussian initial wave packet Equation (20) with $\sigma=0.5$.

\section{Conclusions and Discussion}

In this article, we considered the space-fractional Schrödinger equation with the Riesz space-fractional derivative in the presence of the linear potential. The general solution to the 1D Schrödinger equation in momentum space has been obtained in closed form, which allows the determination of other measurable quantities such as the spatial moments. Furthermore, we investigated the time evolution of a Cauchy wave packet in detail. Besides the 1D Schrödinger equation we also addressed the case of two spatial dimensions. The obtained equations have been illustrated and verified by comparisons with an independent recently proposed numerical scheme. Consequently, the derived equations are therefore also useful for verification of other numerical approaches which are designed for solving the fractional Schrödinger equation. Besides the space-fractional time-dependent Schrödinger equation solutions to the associated time-independent counterpart are also of importance. Concerning the relationship between the space-fractional time-dependent and time-independent Schrödinger equation we refer to the article [26].

Author Contributions: Both authors have worked together in order to prepare the present manuscript.

Conflicts of Interest: The authors declare no conflict of interest.

\section{References}

1. Laskin, N. Fractional quantum mechanics. Phys. Rev. E 2000, 62, 3135.

2. Laskin, N. Fractional Schrödinger equation. Phys. Rev. E 2002, 66, 056108. 
3. Metzler, R.; Klafter, J. The random walk's guide to anomalous diffusion: A fractional dynamics approach. Phys. Rep. 2000, 339, 1-77.

4. Mainardi, F.; Luchko, Y.; Pagnini, G. The fundamental solution of the space-time fractional diffusion equation. Fract. Calc. Appl. Anal. 2001, 4, 153-192.

5. Metzler, R.; Klafter, J. The restaurant at the end of the random walk: Recent developments in the description of anomalous transport by fractional dynamics. J. Phys. A Math. Gen. 2004, 37, R161.

6. Debnath, L.; Bhatta, D. Integral Transforms and Their Applications; CRC Press: New York, NY, USA, 2014.

7. Sandev, T.; Metzler, R.; Tomovski, Z. Correlation functions for the fractional generalized Langevin equation in the presence of internal and external noise. J. Math. Phys. 2014, 55, 023301.

8. Machida, M. The time-fractional radiative transport equation-Continuous-time random walk, diffusion approximation, and Legendre-polynomial expansion. Available online: http://arxiv.org/abs/1602.05382 (accessed on 25 April 2016).

9. Mathai, A.M.; Saxena, R.K.; Haubold, H.J. The H-Function: Theory and Applications; Springer Verlag: New York, NY, USA, 2010.

10. Zhang, Y.; Liu, X.; Belić, M.R.; Zhong, W.; Zhang, Y.; Xiao, M. Propagation Dynamics of a Light Beam in a Fractional Schrödinger Equation. Phys. Rev. Lett. 2015, 115, 180403.

11. Zhang, Y.; Zhong, H.; Belić, M.R.; Ahmed, N.; Zhang, Y.; Xiao, M. Diffraction-free beams in fractional Schrödinger equation. Available online: http://arxiv.org/abs/1512.08671 (accessed on 25 April 2016).

12. Zhang, Y.; Zhong, H.; Belić, M.R.; Zhu, Y.; Zhong, W.; Zhang, Y.; Christodoulides, D.N.; Xiao, M. $\mathcal{P} \mathcal{T}$ symmetry in a fractional Schrödinger equation. Laser Photonics Rev. 2016, doi:10.1002/lpor.201600037.

13. Longhi, S. Fractional Schrödinger equation in optics. Opt. Lett. 2015, 40, 1117-1120.

14. Robinett, R.W. Quantum mechanical time-development operator for the uniformly accelerated particle. Am. J. Phys. 1996, 64, 803-807.

15. Guedes, I. Solution of the Schrödinger equation for the time-dependent linear potential. Phys. Rev. A 2001, 63, 034102.

16. Plastino, A.R.; Tsallis, C. Nonlinear Schroedinger equation in the presence of uniform acceleration. J. Math. Phys. 2013, 54, 041505.

17. Sandev, T.; Petreska, I.; Lenzi, E.K. Time-dependent Schrödinger-like equation with nonlocal term. J. Math. Phys. 2014, 55, 092105.

18. Luchko, Y. Fractional Schrödinger equation for a particle moving in a potential well. J. Math. Phys. 2013, 54,012111

19. Al-Saqabi, B.; Boyadjiev, L.; Luchko, Y. Comments on employing the Riesz-Feller derivative in the Schrödinger equation. Eur. Phys. J. Spec. Top. 2013, 222, 1779-1794.

20. Luchko, Y. Wave-diffusion dualism of the neutral-fractional processes. J. Comput. Phys. 2015, 293, 40-52.

21. Adams, D.; Hedberg, L. Function Spaces and Potential Theory; Springer-Verlag: Berlin, Germany, 1999.

22. Popolizio, M. A matrix approach for partial differential equations with Riesz space fractional derivatives. Eur. Phys. J. Spec. Top. 2013, 222, 1975-1985.

23. Dubbeldam, J.L.A.; Tomovski, Z.; Sandev, T. Space-Time Fractional Schrödinger Equation With Composite Time Fractional Derivative. Fract. Calc. Appl. Anal. 2015, 18, 1179-1200.

24. Debnath, L. Recent applications of fractional calculus to science and engineering. Int. J. Math. Math. Sci. 2003, 2003, 3413-3442.

25. Luchko, Y. Fractional wave equation and damped waves. J. Math. Phys. 2013, 54, 031505.

26. Dong, J. Scattering problems in the fractional quantum mechanics governed by the 2D space-fractional Schrödinger equation. J. Math. Phys. 2014, 55, 032102.

(C) 2016 by the authors; licensee MDPI, Basel, Switzerland. This article is an open access article distributed under the terms and conditions of the Creative Commons Attribution (CC-BY) license (http://creativecommons.org/licenses/by/4.0/). 